\title{
Macroscopic Fundamental Diagram based Road Network Characteristics Identification and Simulation
}

\author{
Yi $\mathrm{Yu}^{1 *}$, Liang Wang ${ }^{1}$, Xianglun $\mathrm{Mo}^{2 *}$, Yao $\mathrm{Yu}^{3}$ and Mei liu ${ }^{4}$ \\ ${ }^{1}$ Shanghai Jiuxuan Engineering Technology Co., Ltd.,201000 Shanghai, China \\ 2. China University of Mining and Technology, School of mines, 221116, China \\ ${ }^{3}$ Shanghai Maritime University, College of Transport and Communications, 201306 Shanghai, China \\ ${ }^{4}$ Shanghai Maritime University, China Institute of FTZ Supply Chain, 201306 Shanghai, China
}

\begin{abstract}
As an inherent property of the road network, macroscopic fundamental diagram (MFD) method can effectively describe the traffic status of the urban roads and identify the relationship among key factors, such as traffic flow and occupancy. Currently, using MFD is easily affected by various network inner factors including topology and road density, so in this paper we propose a method to identify inner characteristic of road network and do a series of comparisons under different scenarios with fixed traffic input circumstance. The differential impact of data collector setting locations are discussed with a aim to reveal the respective location setting suitable for various networks conditions in initial; then road topology and density are designed in road network and simulated MFD performances with flow equilibrium affections. It is shown as the dispersion decreasing of link length or road density of network, the network exhibits better operation efficiency so as to increase the output of link flow and the dissipative ability of the road network. Meanwhile, the equivalent of entrances and exits is proved as another important factor has same impact on MFD.
\end{abstract}

\section{Introduction}

In metropolis, traffic jam and motor vehicle's pollution have become critical problems even threaten people's daily life. Traffic control is viewed as one of effective ways to resolve these problems. However, the current urban traffic management mainly depends on the data collection from road scale and accordingly makes a decision in terms of microscopic traffic control principle which often lead to congestion transfer. Considering from network integration control, macroscopic fundamental diagram (MFD) ${ }^{[1-2]}$ is well used and already proved to be an effective way to describe the network operation status from macroscopic perspective and also can identify the inner relationship among elements which constitute of urban road network.

\section{Literature reviews}

The prototype of the macroscopic fundamental diagram (MFD) was first proposed by Ardekani and Herman ${ }^{[3]}$ according to actual data collection and found that the average flow speed is related to vehicle accumulation through a linear relationship, however the maximum observed accumulation only reach to 30 vehicles/Miles. Based on this research, Geroliminis and Daganzo ${ }^{[2]}$ analyzed a more congested network, central part of Yokoham. In this network, it was observed that MFD can be defined at a low level of observational scattering.
MFD theory can describe traffic conditions with fewer parameters, without requiring a detailed description of traffic conditions.

For better control in urban regional, MFD needs to divide road network. A series of studies have been conducted and influencing factors also been proposed. In terms of the influencing factors of the MFD, the main research factors are classified by traffic conditions, road conditions, control conditions and selection behavior. In road conditions, the main research factors are the nature of the dedicated roads and key road which loading massive flow, also with their length and topology of network $^{[4-6]}$. And Buission ${ }^{[7]}$ used detectors data in Toulouse, a medium-sized French city, to explore the impact of different road network structures on MFD. Studies have shown that urban normal road networks and high-speed road networks exhibited different MFD shapes and hysteresis. Detector setting location has a significant affection of the maximum flow rate. However, there still exist limitations on Buission's research, such as data integrity, road assumptions and lack of quantitative analysis. Besides, the different formation and dissipation of the congestion phenomenon will have a greater impact on the hysteresis phenomenon of the MFD. It is necessary to quantify the interrelation between different inner elements and MFD performance ${ }^{[8-10]}$. Therefore, this paper considers various network features including link length, network topology

\footnotetext{
*Corresponding author: yyleno@126.com
} 
and link density to calculate and explain their impact on MFD in detail.

\section{Detectors setting location analysis}

\subsection{Road network design}

In order to display the characteristics of different road networks more intuitively, we propose a grid network. This benchmark road network consists of 9 intersections, each of which has four directions, which are bidirectional six lanes. Three lanes in one direction indicate left, straight and right turn from left to right respectively with a period of 80 seconds. And length of each road section is 300 meters. as shown in Fig 1.

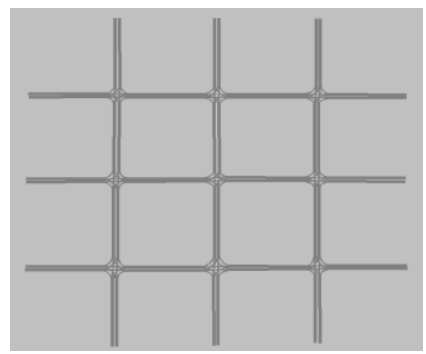

Figure.1 Sample of road network include 9 nodes

Here we set vehicles input value is large enough to make the whole network get a saturation status. And the flow is changed every 600 seconds for a total of 15 phases, distribution as shown in Fig. 2.

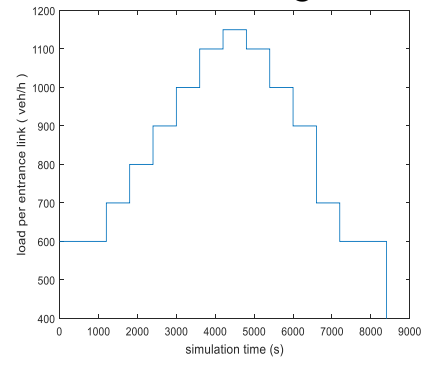

Fig.2 Vehicles loading distribution

\subsection{Detectors setting analysis}

The relationship between the occupancy rate and traffic flow are observed to determine the location of the detector by MFD. We choose the most loading flow link (MLF) and least loading link (LLL) in the whole network. And detectors are set at the exit of the upstream intersection, at the centre of the link and at the entrance of the downstream intersection respectively.

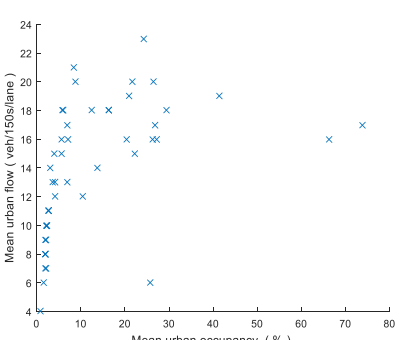

(a)

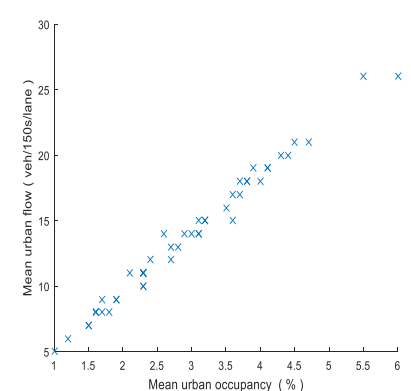

(c)

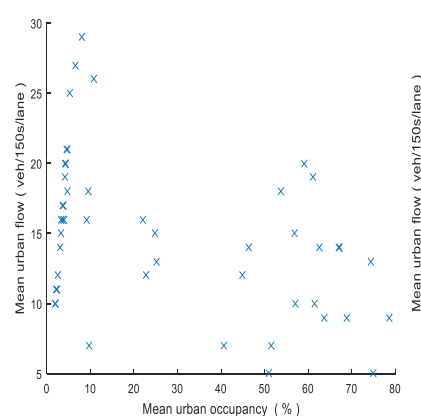

(e)

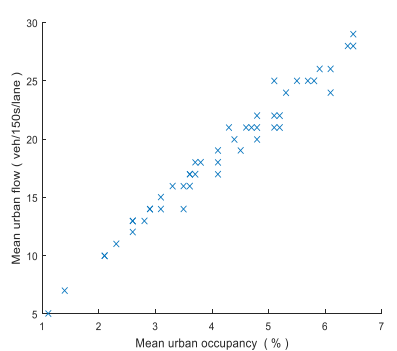

(b)

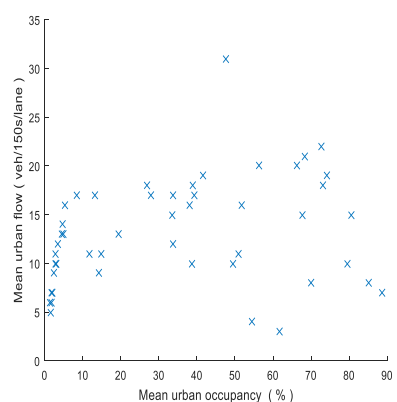

(d)

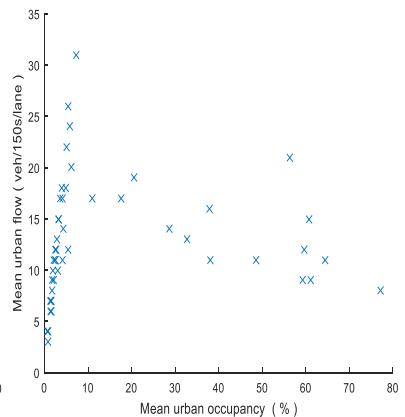

(f)

Figure.3 MFD under different detectors setting locations: (a) LLL near the entrance of intersection; (b) middle of LLL; (c) LLL far from the entrance of intersection; (d) MLF near the intersection entrance; (e) middle of MLF; (f) MLF far from the entrance of intersection)

It can be observed that detectors on LLL shows large number of scattered points as well as occupancy rate when near the entrance of intersection as Fig.4a shows; meanwhile, there exists a linear upward trend in Fig. $3 \mathrm{~b}$ and Fig.3c. There are few scattered spots with small occupancy rate when we choose LLL. On the contrary, MLF shows a large number of scattered spots and high occupancy rates in Fig.3d to Fig.3f. Furthermore, the results implied that if detector setting closer to intersection, the scattered phenomenon is more obviously. And the distance from the entrance to the intersection presents a linear upward trend. Therefore, in order to obtain accurate MFD (decreasing trend due to congestion), more detectors should be set closer to the entrance of intersection.

\subsection{Data collection}

Simulation time is set 9000 seconds, in which first 1800 seconds are warming process. There are 48 sets of simulated data are obtained. Here we define the average 
flow and average occupancy as equation (1) and (2) shows respectively.

$$
\begin{gathered}
Q=\frac{1}{N} \sum_{\mathrm{i}=1}^{N}\left(\frac{1}{N_{\mathrm{i}}} \sum_{j=1}^{N_{i}} q_{i j}\right) \\
O=\frac{1}{N} \sum_{\mathrm{i}=1}^{N}\left(\frac{1}{N_{i}} \sum_{j=1}^{N_{i}} \boldsymbol{O}_{i j}\right)
\end{gathered}
$$

Where $i$ represents the number of the detector; $N_{i}$ is the number of detectors in the detector group $N$ that are working normally; $N$ is the total number of detectors set in the road network; $q$ and $o$ are the flow rates and occupancy rates detected by the detector; $Q$ is the average traffic flow of the road network; $O$ is the average occupancy of the road network.

This paper choose occupancy rate to represent the relationship with the traffic flow instead of density because 1) vehicles' length in actual road network cannot be accurately observed; 2) the proportion of trucks among the flow is $2 \%$. So using occupancy rate would be a good estimation method of the density.

\section{Simulation results}

\section{1 link-based analysis}

In Table 1, it showed four different scenrios of network characteristics to compare their MFDs and dig impact factor would affect on flow equilibrium. Among them, scenrio a as a benchmark, and simulated MFD results shown as Fig.4.

Table 1. Road network form

\begin{tabular}{|c|c|}
\hline No. & Road network characteristics scenarios \\
\hline (a) & Benchmark road network \\
\hline (b) & Reduced total length of links (1 direction) \\
\hline (c) & Reduced total length of links (2 direction) \\
\hline (d) & Increased dispersion of link length \\
\hline
\end{tabular}

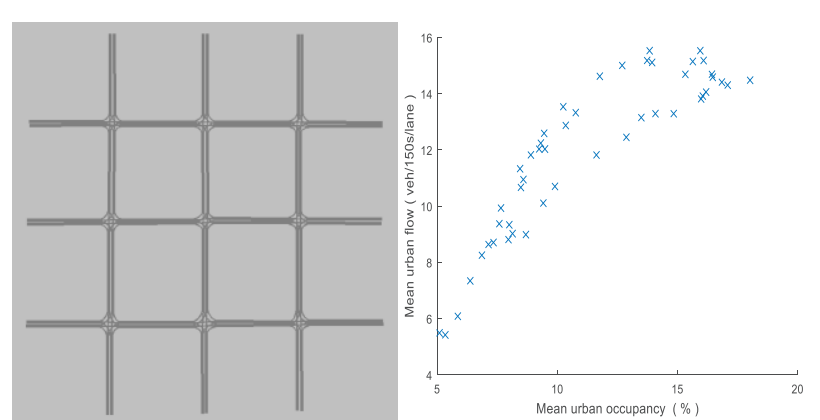

(a)

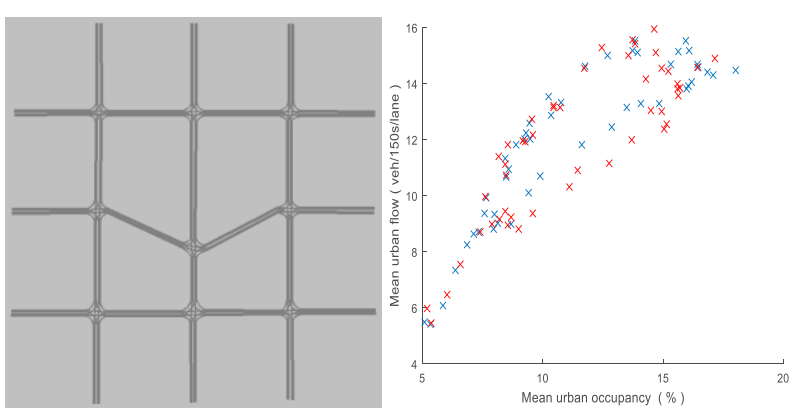

(b)

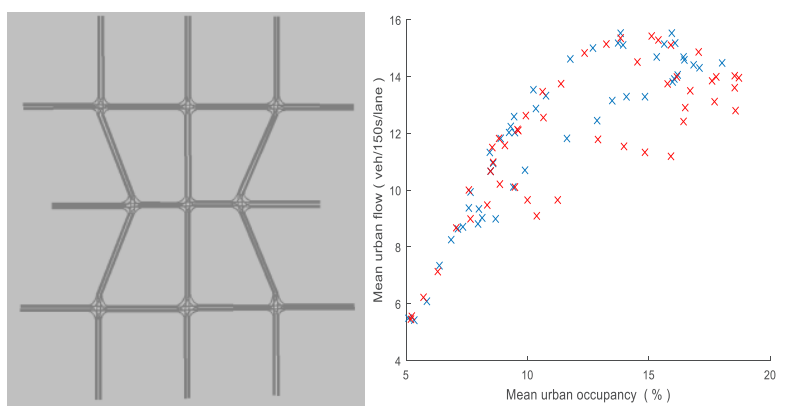

(c)
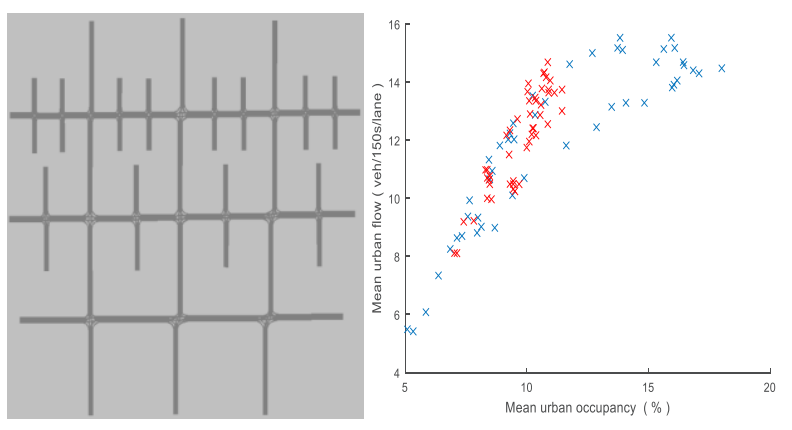

(d)

Figure.4 MFDs under four scenarios (blue cross is benchmark in (a) and red cross is the corresponding MFD in its own scenario)

According to Fig. $4 \mathrm{~b}$ and Fig.4c, there is no major change in the upper curve of the MFD, however there is a large change in the hysteresis loop. The hysteresis of scenario c has a large change in shape. (b) compared with benchmark (a), the length of the road section in one direction is shortened but the length of the other three directions is increased. In Tab.2, the maximum occupancy rate is decreased by $4.74 \%$, the maximum traffic volume is increased by $2.66 \%$, and the critical occupancy rate is increased by $0.13 \%$. Fig 4 (c) compared with (a), the length of two links are shortened at the intersection of the network centre. The maximum occupancy rate increased by $3.88 \%$, however, the maximum traffic volume decreased by $0.69 \%$, and the critical occupancy rate increased by $0.16 \%$. The maximum traffic volume and key occupancy of (b) have increased slightly, but the extent of change has been too small. Therefore, it is concluded that the effect of the changeable of links length (a certain intersection) on the MFD is not obvious. 
Table 2 Comparison of MFD considering link length

\begin{tabular}{|c|c|c|c|c|c|}
\hline No. & $\begin{array}{c}\text { Maximum } \\
\text { occupancy }\end{array}$ & $\begin{array}{c}\text { Maximum } \\
\text { occupancy } \\
\text { compared } \\
\text { with a }\end{array}$ & $\begin{array}{c}\text { Maximum } \\
\text { production } \\
\text { compared } \\
\text { with a }\end{array}$ & $\begin{array}{c}\text { Critical } \\
\text { occupancy } \\
\text { rate }\end{array}$ & $\begin{array}{c}\text { Critical } \\
\text { occupancy } \\
\text { rate } \\
\text { compared } \\
\text { with a }\end{array}$ \\
\hline a & 18.002 & $/$ & $/$ & 13.817 & $/$ \\
\hline b & 17.149 & $-4.74 \%$ & $2.66 \%$ & 13.835 & $0.13 \%$ \\
\hline c & 18.701 & $3.88 \%$ & $-0.69 \%$ & 13.839 & $0.16 \%$ \\
\hline d & 11.461 & $-36.33 \%$ & $-5.42 \%$ & 10.779 & $-21.99 \%$ \\
\hline
\end{tabular}

In scenario d, it changes more links length aims to reach a greater degree of dispersion. And the results show that the maximum occupancy and critical occupation rate are both greatly reduction. And the degree of utilization of the road network also reduced. When the occupancy rate is small, traffic flow begins to decline. The maximum traffic volume decreased by $5.42 \%$, and the performance of the road network decreased. At the same time, compared with (a), (b), and (c), the performance of the road network decreases when the dispersion of links increases.

\subsection{Density based analysis}

Density-based tests are conducted to compare the average occupancy and average flow performance in MFD. The MFD results in Fig.5 and in Table.3.
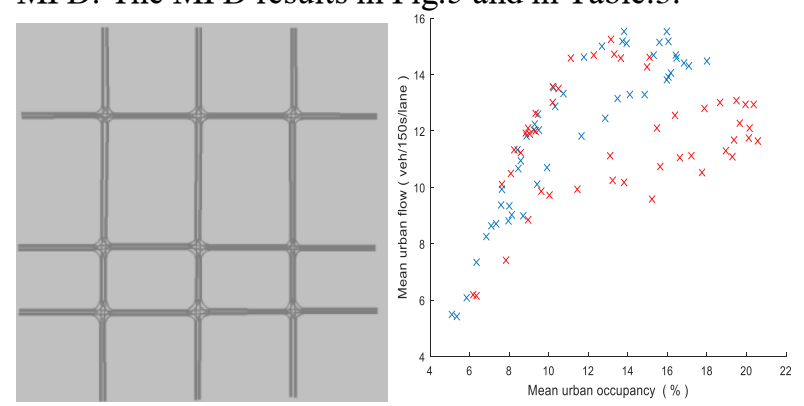

(e)
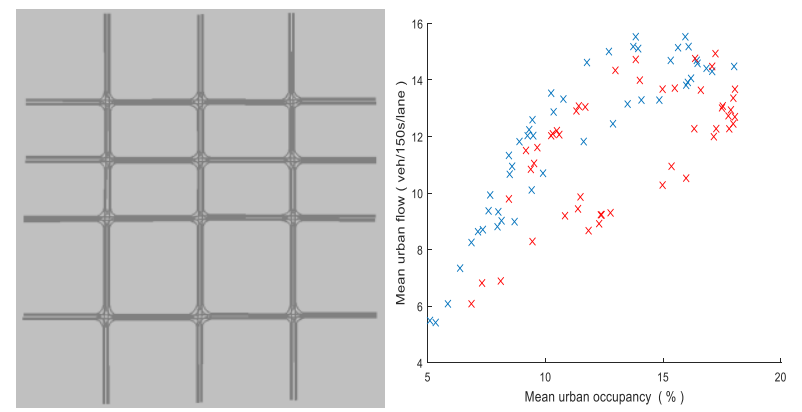

(f)
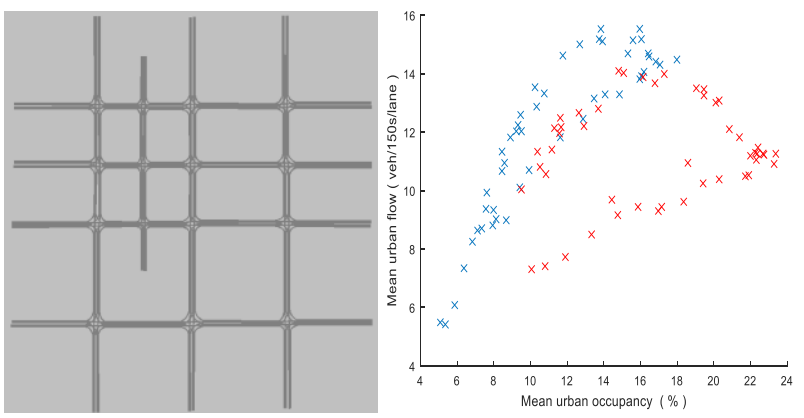

(g)

Figure.5 MFDs under different density conditions (blue cross is benchmark in (a) and red cross is the corresponding MFD in its own scenario)

Table 3 Comparison of MFD considering density

\begin{tabular}{|c|c|c|c|c|c|}
\hline No. & $\begin{array}{c}\text { Maximum } \\
\text { occupancy }\end{array}$ & $\begin{array}{c}\text { Maximum } \\
\text { occupancy } \\
\text { compared } \\
\text { with a }\end{array}$ & $\begin{array}{c}\text { Variation } \\
\text { compared } \\
\text { with a }\end{array}$ & $\begin{array}{c}\text { Critical } \\
\text { occupancy } \\
\text { rate }\end{array}$ & $\begin{array}{c}\text { Critical } \\
\text { occupancy } \\
\text { rate } \\
\text { compared } \\
\text { with a }\end{array}$ \\
\hline $\mathrm{a}$ & 18.002 & $/$ & $/$ & 13.817 & $/$ \\
\hline $\mathrm{e}$ & 20.571 & $14.27 \%$ & $-1.81 \%$ & 13.295 & $-3.78 \%$ \\
\hline $\mathrm{f}$ & 18.037 & $0.19 \%$ & $-3.83 \%$ & 13.841 & $0.17 \%$ \\
\hline $\mathrm{g}$ & 23.379 & $29.87 \%$ & $-9.35 \%$ & 14.786 & $7.01 \%$ \\
\hline
\end{tabular}

From the above three conditions, it can be seen that the unevenness of the road network density will degrade the performance of the road network. Although the critical occupancy rate will increase, the maximum traffic flow will decrease, and the road network performance will decline sharply when the congestion disappears. Therefore, when planning the road network, the density gap between the road segments in the road network should be avoided so that the density can be balanced to obtain a macroscopic fundamental diagram with smaller traffic volume in the hysteresis loop.

\subsection{Numbers of entrances and exits based analysis}

Similarly, numbers of entrances and exits based analysis tests are conducted to compare the average occupancy and average flow performance in MFD. The MFD results in Fig. 6 and in Table.4.

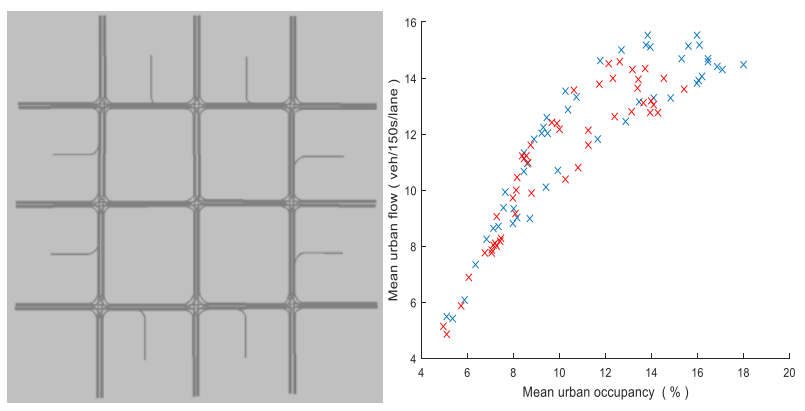

Figure. 6 MFD under change of numbers of entrances and exits (blue cross is benchmark in (a)) 
Table 4 Comparison of key points of MFD under the feature of number of entrances and exits

\begin{tabular}{|c|c|c|c|c|c|}
\hline No. & $\begin{array}{c}\text { Maximum } \\
\text { occupancy }\end{array}$ & $\begin{array}{c}\text { Maximum } \\
\text { occupancy } \\
\text { compared } \\
\text { with a }\end{array}$ & $\begin{array}{c}\text { Maximum } \\
\text { production } \\
\text { compared } \\
\text { with a }\end{array}$ & $\begin{array}{c}\text { Critical } \\
\text { occupancy } \\
\text { rate }\end{array}$ & $\begin{array}{c}\text { Critical } \\
\text { occupancy } \\
\text { rate } \\
\text { compared } \\
\text { with a }\end{array}$ \\
\hline $\mathrm{a}$ & 18.002 & $/$ & $/$ & 13.817 & $/$ \\
\hline $\mathrm{h}$ & 15.349 & $-14.74 \%$ & $-6.11 \%$ & 12.608 & $-8.75 \%$ \\
\hline
\end{tabular}

In (h), as increasing of the number of exports to double the number of entrances on the basis of the benchmark, the MFD is almost enveloping in the baseline MFD. Table 4 also shows that the maximum occupancy rate, critical occupancy rate, and maximum traffic volume have all decreased, and the overall road network performance has dropped. Therefore, ensuring the balance of the number of entrances and exits is also a key factor in improving the performance of the road network.

Table 5 MFD characteristics compare with benchmark

\begin{tabular}{|c|c|c|c|c|}
\hline \multicolumn{2}{|c|}{} & $\begin{array}{c}\text { Maximum } \\
\text { occupancy }\end{array}$ & Variation & $\begin{array}{c}\text { Critical } \\
\text { occupancy }\end{array}$ \\
\hline \multirow{2}{*}{$\begin{array}{c}\text { links } \\
\text { length }\end{array}$} & $\mathrm{b}$ & $-4.74 \%$ & $2.66 \%$ & $0.13 \%$ \\
\cline { 2 - 5 } & $\mathrm{c}$ & $3.88 \%$ & $-0.69 \%$ & $0.16 \%$ \\
\hline & $\mathrm{d}$ & $-36.33 \%$ & $-5.42 \%$ & $-21.99 \%$ \\
\hline \multirow{4}{*}{ density } & $\mathrm{e}$ & $14.27 \%$ & $-1.81 \%$ & $-3.78 \%$ \\
\cline { 2 - 5 } & $\mathrm{f}$ & $0.19 \%$ & $-3.83 \%$ & $0.17 \%$ \\
\cline { 2 - 5 } & $\mathrm{g}$ & $29.87 \%$ & $-9.35 \%$ & $7.01 \%$ \\
\hline $\begin{array}{c}\text { entrances } \\
\text { or exits } \\
\text { numbers }\end{array}$ & $\mathrm{h}$ & $-14.74 \%$ & $-6.11 \%$ & $-8.75 \%$ \\
\hline
\end{tabular}

From the above figures and statistic in Tab.5, either total link length or density has significant impact on the curve of the MFD with different peak value. Road network performance would sharply decreases when the dispersion of network density at a high level. Therefore, to balance the road network characteristics is an effective way to improve the running performance of the road network. It should be throughout taken into account when make a network planning.

\section{Conclusion}

This paper discusses influential factors impact on the performance of macroscopic fundamental diagram (MFD) according to a series of simulated scenarios on the basis of comparisons on dispersion of hysteresis phenomena and values of average flow and average occupancy.

From the above simulated results in section 4 , the characteristics of the road network have a great impact on the MFD, such as link length, link density and numbers of entrances and exits. The inequality of road length and road network density would change the shape of the MFD to a great extent and lower the performance of the whole network.

The discreteness of link length is important to the performance of MFD. The unequal distribution of length usually leads to a sharp decline of the performance of the road network, which does not reach the maximum value of output flow. Considering a small road network density scenario, the output flow is decreasing, and the network cannot be fully used. This uneven distribution of road network density will also have an impact on the MFD and degrade the performance of traffic running. In addition, on the case of the number of exits is greater than the number of entrances, the integrate MFD is wrapped than the basic MFD as benchmark scenario (a) shows. On the contrary, when the number of exits is less than the number of entrance, the road occupancy rate would significantly raise.

The dispersion of hysteresis indicates congestion dissipating capacity of the road network. The smaller the degree of dispersion, the more the performance of the network can be used through congestion dissipation. If the distribution of network density is uneven, congestion in lower density areas will quickly dissipate. However, due to the existence of signal phase in high density areas, jam cannot be alleviated in a short period, and a large lag discrete empirical risk value is obtained. In addition, the high degree of dispersion of the link length would lead to exacerbate the lag phenomenon to a certain extent. This phenomenon will cause the running status deterioration of the road network during the process of dissipation. Theoretically, the number of exits increment will accelerate the dissipation of congestion, however the effect is not obvious. In traffic planning process, link length and the unevenness of network density should be taken into consideration to minimize the un-equilibrium, so as to reach the maximum road network loading capacity and get optimal operation status.

\section{Acknowledgement}

This study is supported by the "National Natural Science Foundation of China" (grant no. 61603247)

\section{Reference}

1. Geroliminis N, Daganzo C F. Existence of urbanscale macroscopic fundamental diagrams:590 Some experimental findings[J]. Transportation Research Part B Methodological, 2008, 591 42(9):759-770.

2. Geroliminis N, Daganzo C F. Macroscopic modeling of traffic in cities[C]. Transportation Research Board 86th Annual Meeting. 2007.

3. Ardekani S, Herman R. Urban Network-Wide Traffic Variables and Their Relations[M]. INFORMS, 1987.

4. Ma Wanjing, Liao Dabin. Macroscopic fundamental diagram of network traffic flow: retrospect and prospect[J]. Journal of Wuhan University of Technology (Transportation Science and Engineering Edition), 2014, 38(6):1226-1233.

5. $\mathrm{Xu}$ Feifei, He Zhaocheng, Sha Zhiren. Impact Analysis of Traffic Management Measures on Macroscopic fundamental diagram of Road 
Network[J]. Journal of Transportation Systems Engineering and, 2013, 13(2):185-190.

6. Zhu Lin, Yu Lei, Song Guohua. Research on Macroscopic Traffic Status and Influencing Factors of Road Network Based on MFD[J]. Journal of South China University of Technology (Natural Science Edition), 2012, 40(11):138-146.

7. Buisson $\mathrm{C}$, Ladier C. Exploring the Impact of Homogeneity of Traffic Measurements on the Existence of Macroscopic Fundamental Diagrams[J]. Transportation Research Record Journal of the Transportation Research Board, 2009, 137(2124):127-136.

8. Courbon T, Leclercq L. Cross-comparison of Macroscopic Fundamental Diagram Estimation Methods[J]. Procedia - Social and Behavioral Sciences, 2011, 20(6):417-426.

9. Yildirimoglu M, Ramezani M, Geroliminis N. Equilibrium analysis and route guidance in largescale networks with MFD dynamics[J]. Transportation Research Part C Emerging Technologies, 2015, 59:404-420.

10. Saberi M, Mahmassani H S. Exploring Properties of Networkwide Flow-Density Relations in a Freeway Network[J]. Transportation Research Record Journal of the Transportation Research Board,2012, 2315(2315):153-163. 